\title{
Positron emission tomography in the diagnosis of Whipple's endocarditis: a case report
}

\author{
Sarah-Lyne Jos ${ }^{1}$, Emmanouil Angelakis ${ }^{1 *}$, Thierry Caus $^{2}$ and Didier Raoult ${ }^{1}$
}

\begin{abstract}
Background: Whipple's disease is a systemic infection that sometimes is associated with cardiac manifestations. The diagnosis of Tropheryma whipplei endocarditis is still the result of chance because there are no diagnostic criteria and clinical signs are often those of cardiac disease rather than infection.

Case presentation: Culture-negative endocarditis was suspected in a non-febrile 77-year-old French woman from North France with a history of a graft replacement 4 years prior. Positron emission tomography revealed intense fluorodeoxyglucose uptake around the metal ring of the aortic graft. The valve was replaced, and T. whipplei was detected in a valve sample by molecular assays. Immunohistochemical staining of the valve for T. whipplei was also positive.

Conclusion: The localization of infectious foci by positron emission tomography and systematically testing valve specimens for T. whipplei are promising for diagnosing Whipple's disease.
\end{abstract}

Keywords: Positron emission tomography, Tropheryma whipplei, Culture-negative endocarditis

\section{Background}

Cardiovascular disease is the leading cause of death in the United States and worldwide [1]. Blood culturenegative endocarditis accounts for $2.5 \%$ to $31 \%$ of all cases of endocarditis [2]. Blood culture-negative endocarditis is a severe and difficult-to-diagnose disease, but our understanding of it has greatly improved over the past 2 decades [2]. Tropheryma whipplei, the causative agent of Whipple's disease has been indicated as an agent of blood culture-negative endocarditis [3]. T. whipplei endocarditis differs from classic Whipple's disease, which primarily affects the gastrointestinal system. The bacterium can also cause localized chronic infections such as spondylodiscitis, meningoencephalitis, uveitis, and pneumonia. T. whipplei endocarditis is an emerging clinical entity mostly observed in middle-aged and older men with arthralgia [3]. The diagnosis of $T$. whipplei endocarditis is based on molecular assays of surgically obtained heart valves [3]. Positron emission tomography (PET) is a promising tool for the identification of infectious foci, especially in culture-negative

\footnotetext{
* Correspondence: angelotasmanos@msn.com

'URMITE CNRS-IRD 198 UMR 6236, Aix Marseille Université, Faculté de Médecine et de Pharmacie, 27 Bd Jean Moulin, 13385 Marseille, France

Full list of author information is available at the end of the article
}

infected cardiovascular devices [4]. PET scanning has higher sensitivity than computed tomography (CT) for the evaluation of the extent and localization of infections [4]. We report one case of a culture-negative endocarditis localized by PET and diagnosed as T. whipplei endocarditis by molecular assays and histology.

\section{Case presentation}

A non-febrile 77-year-old French woman from North France was admitted to the hospital for faintness and neurological deficit with right hemiplegia and aphasia. The patient had a history of a graft replacement of her aortic valve 4 years before. Laboratory values revealed increased C-reactive protein and white blood cell count. Liver enzyme levels were normal. Brain magnetic resonance imaging (MRI) revealed two recent ischemic areas and CT scanning showed linear hypodensity in the posterior part of the spleen and two hypodense areas of the cortex of the right kidney. Based on the numerous ischemic zones, a cardiac cause was suspected. Transesophageal echocardiogram showed diffuse thickening of the bioprosthesis; mobile, high-potential-embolic, bioprosthesis stenosis; and intra-prosthetic aortic insufficiency. PET scan revealed intense fluorodeoxyglucose uptake around the metal ring of the aortic graft (Figure 1). The 


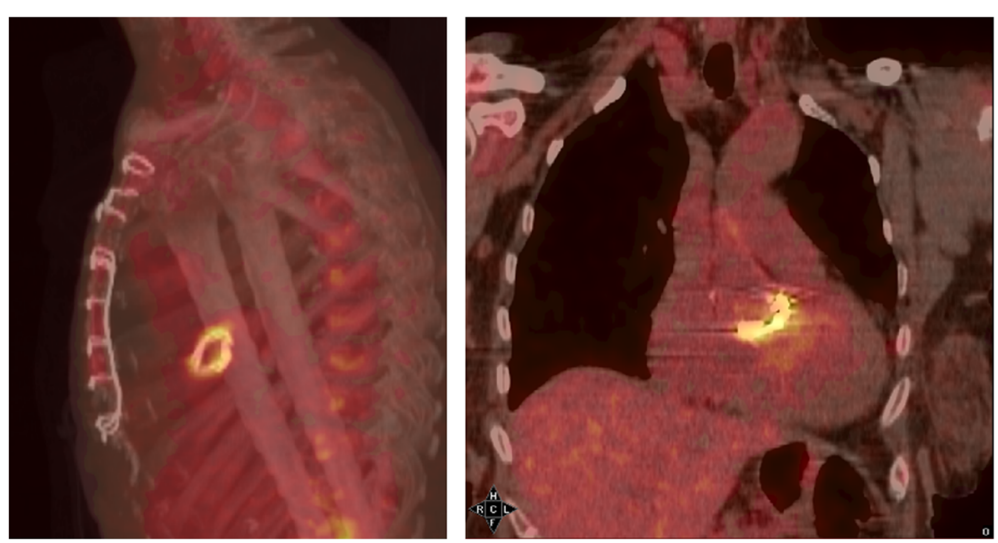

Figure 1 Fluorodeoxyglucose uptake around the metal ring of the aortic graft revealed by PET scan.

valve bioprosthesis was replaced, and treatment with amoxicillin and clavulanic acid (12 g/day) and gentamycin (120 mg 2 times per day) was started. Gram staining and standard cultures of the valve and blood were all negative. A valve sample was sent to our reference center in Marseille. DNA was extracted from this sample using a QIAamp tissue kit (Qiagen, Hilden, Germany) according to the manufacturer's recommendations. Then, DNA extracts were tested by qPCR for the presence of Tropheryma whipplei, Coxiella burnetti, Bartonella sp., Staphylococcus aureus, Enterococcus faecalis, Enterococcus faecium, Mycoplasma pneumonia, Streptococcus oralis, Streptococcus gallolyticus, Escherichia coli and fungi [5]. All assays were negative except those for T. whipplei. A second $\mathrm{qPCR}$ assay targeting repeated sequences of $T$. whipplei $[6,7]$ confirmed the diagnosis. In addition, PCR amplification and sequencing of the 16S rRNA gene [5] was also positive for T. whipplei. Genotyping was performed as previously described [8] and showed that this T. whipplei was genotype 16. Immunohistochemical staining of the valve for T. whipplei was positive (Figure 2) [9]. Culturing of the valve was negative. A course of $200 \mathrm{mg}$ oral doxycycline once per day with $200 \mathrm{mg}$ hydroxychloroquine three times per day for
18 months was introduced. The diagnosis for this patient was T. whipplei endocarditis in the context of a bioprosthetic aortic valve.

\section{Discussion}

We report a case of T. whipplei endocarditis localized using PET scanning. The diagnosis of Whipple's disease was then established by molecular assays and histology on the valve bioprosthesis. Infective endocarditis is associated with poor prognosis despite improvements in medical and surgical therapies [4]. Although the first description of T. whipplei endocarditis was made approximately 15 years ago, diagnosing this disease remains difficult because clinical signs are often those of cardiac disease rather than infection [3,10]. The first case was detected by chance when a broad-spectrum PCR was systematically applied to heart valve specimens [10]. PET scanning has been used to detect periprosthetic valve abscesses even in cases in which transesophageal echocardiography and transthoracic echocardiography were normal or doubtful, particularly in cases of prosthetic valve infections, in which results of initial echocardiography are not useful in 30\% of cases [4]. The localization of infectious foci by PET had

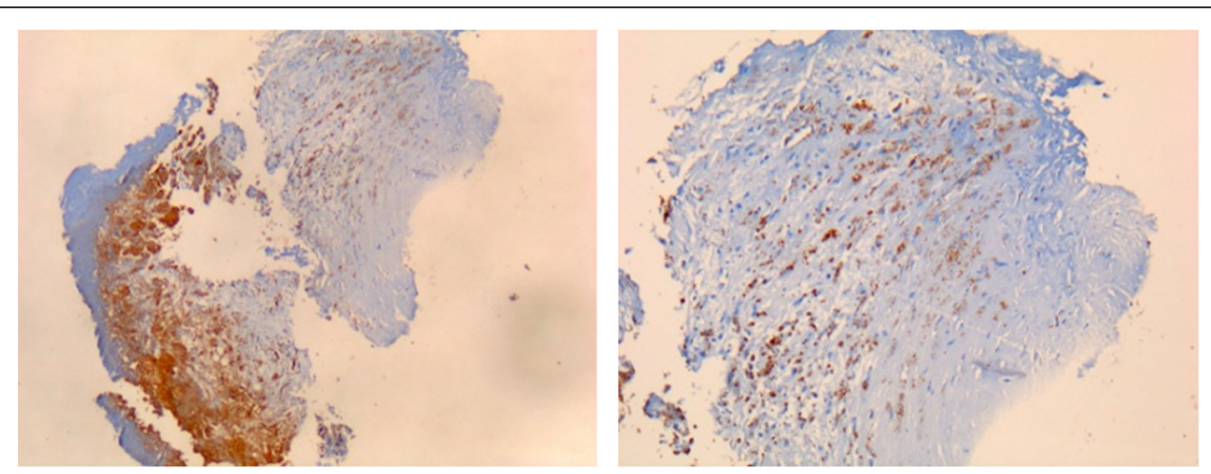

Figure $2 T$. whipplei-positive immunohistochemical staining of the valve sample. 
previously resulted in the diagnosis of only two cases of Whipple's disease [11,12] and has been used to detect periprosthetic valve abscesses even in cases in which transesophageal echocardiography and transthoracic echocardiography were normal or doubtful [4]. In our case, PET scanning was especially valuable in the early diagnosis of $T$. whipplei endocarditis because it identified uptake at the graft replacement of the aortic valve, indicating an infection.

Blood culture-negative endocarditis accounts for $2.5 \%-31 \%$ of all cases of endocarditis. T. whipplei endocarditis is an emerging clinical entity mostly observed in middle-aged and older men with arthralgia [3]. Indeed, the disease occurs mainly in white men who are $\sim 50$ years of age with cardiac manifestations such as heart failure, acute ischemic stroke, and peripheral arterial embolism [3]. T. whipplei endocarditis is a frequent pathogen among cases of endocarditis, but its diagnosis is still the result of chance because there are no diagnostic criteria and clinical signs are often those of cardiac disease rather than infection $[3,13]$. In heart valves, $T$. whipplei is surrounded by an inflammatory process and inside the macrophages [3]. T. whipplei-infected heart valves show the typical histologic features of infective endocarditis: vegetations, inflammatory infiltrates, and valvular destruction. The diagnosis of T. whipplei endocarditis is based on molecular assays of surgically obtained heart valves [3]. The performance of repeat PCR for $T$. whipplei on blood specimens is a major criterion in the Duke classification for endocarditis.

Genotyping revealed that the valve was infected by $T$. whipplei genotype 16. T. whipplei genotyping has shown high genetic diversity unrelated to pathogenicity [14]. $T$. whipplei genotype 16 has been detected in the cerebrospinal fluid of a patient with neurological Whipple's disease in Germany and in the synovial fluid of a patient with classic Whipple's disease [8]. Moreover, it has been detected in the gastric juice of a patient without clinical manifestations from Switzerland [8]. In Europe, T. whipplei genotype 3 is the most common genotype and appears to be epidemic and specific to France, Switzerland, and Italy [8]. The second most common genotype in Europe is the genotype 1 , which is endemic and mainly observed in central Europe.

\section{Conclusions}

We report a case that illustrates the usefulness of ${ }^{18}$ FDG-PET/CT for diagnosis of T. whipplei infectious endocarditis in a patient with a graft replacement of the aortic valve. The localization of infectious foci by PET scanning and the systematic testing of valve specimens for T. whipplei are promising, and these procedures can be performed in patients of all ages by adjusting the dose of ${ }^{18} \mathrm{FDG}$ to the weight of the patient. Thus, we believe that
T. whipplei should be considered in the diagnosis of culture-negative endocarditis and that PET scanning might be helpful in the diagnosis of T. whipplei infectious endocarditis, although it will not replace clinical evaluation, laboratory tests, and echocardiography.

\section{Consent}

Written informed consent was obtained from the patient for publication of this Case Report and any accompanying images. A copy of the written consent is available for review by the Editor-in-Chief of this journal.

\section{Abbreviations}

PET: Positron emission tomography; MRI: Magnetic resonance imaging; $\mathrm{CT}$ : Computed tomography.

\section{Competing interests}

The authors declare that they have no competing interests

\section{Authors' contributions}

SLJ collected the data and wrote the manuscript. EA analyzed the data and wrote the manuscript. TC was involved with management of the case. DR wrote and was responsible for the critical revision and final approval of the paper. All of the authors read and approved the final manuscript.

\section{Author details}

'URMITE CNRS-IRD 198 UMR 6236, Aix Marseille Université, Faculté de Médecine et de Pharmacie, 27 Bd Jean Moulin, 13385 Marseille, France. ${ }^{2}$ INSERM, ERI-12 (EA 4292), University of Picardie, Department of Cardiac Surgery, University Hospital Amiens, Avenue René Laënnec - Salouël, 80054 Amiens, France.

Received: 12 November 2014 Accepted: 19 February 2015

Published online: 26 February 2015

\section{References}

1. Santulli G. Epidemiology of cardiovascular disease in the $21^{\text {st }}$ century: updated numbers and updated facts. J Cardiovascular Disease. 2013;1:1-2.

2. Brouqui $P$, Raoult D. Endocarditis due to rare and fastidious bacteria. Clin Microbiol Rev. 2001;14:177-207.

3. Fenollar F, Celard M, Lagier JC, Lepidi H, Fournier PE, Raoult D. Tropheryma whipplei endocarditis. Emerg Infect Dis. 2013;19:1721-30.

4. Thuny F, Gaubert JY, Jacquier A, Tessonnier L, Cammilleri S, Raoult D, et al. Imaging investigations in infective endocarditis: current approach and perspectives. Arch Cardiovasc Dis. 2013;106:52-62.

5. Safont M, Angelakis E, Richet $H$, Lepidi H, Fournier PE, Drancourt M, et al. Bacterial lymphadenitis at a major referral hospital in France from 2008 to 2012. J Clin Microbiol. 2014;52:1161-7.

6. Fenollar F, Laouira S, Lepidi H, Rolain JM, Raoult D. Value of Tropheryma whipplei quantitative polymerase chain reaction assay for the diagnosis of Whipple disease: usefulness of saliva and stool specimens for first-line screening. Clin Infect Dis. 2008;47:659-67.

7. Angelakis E, Fenollar F, Lepidi H, Birg ML, Raoult D. Tropheryma whipplei in the skin of patients with classic Whipple's disease. J Infect. 2010;61:266-9.

8. Li W, Fenollar F, Rolain JM, Fournier PE, Feurle GE, Muller C, et al. Genotyping reveals a wide heterogeneity of Tropheryma whipplei. Microbiology. 2008;154:521-7.

9. Lepidi H, Fenollar F, Dumler JS, Gauduchon V, Chalabreysse L, Bammert A, et al. Cardiac valves in patients with Whipple endocarditis: microbiological, molecular, quantitative histologic, and immunohistochemical studies of 5 patients. J Infect Dis. 2004;190:935-45.

10. Goldenberger D, Kunzli A, Vogt P, Zbinden R, Altwegg M. Molecular diagnosis of bacterial endocarditis by broad-range PCR amplification and direct sequencing. J Clin Microbiol. 1997;35:2733-9.

11. Andre MF, Delevaux I, Cachin F, Fenollar F, Raoult D, Maublant J, et al. 18 F-fluorodeoxyglucose positron emission tomography in Whipple's disease. Scand J Gastroenterol. 2006;41:1491-2. 
12. Dumarey N, Egrise D, Blocklet D, Stallenberg B, Remmelink M, del Marmol V, et al. Imaging infection with 18 F-FDG-labeled leukocyte PET/CT: initial experience in 21 patients. J Nucl Med. 2006;47:625-32.

13. Geissdorfer W, Moos V, Moter A, Loddenkemper C, Jansen A, Tandler R, et al. High frequency of Tropheryma whipplei in culture-negative endocarditis. J Clin Microbiol. 2012;50:216-22.

14. Keita AK, Bassene H, Tall A, Sokhna C, Ratmanov P, Trape JF, et al. Tropheryma whipplei: a common bacterium in rural Senegal. PLoS Negl Trop Dis. 2011;5:e1403.

Submit your next manuscript to BioMed Central and take full advantage of:

- Convenient online submission

- Thorough peer review

- No space constraints or color figure charges

- Immediate publication on acceptance

- Inclusion in PubMed, CAS, Scopus and Google Scholar

- Research which is freely available for redistribution 